\title{
Application of Bayesian geostatistical modeling for the assessment of risk for child mortality during the 2008 earthquake in Wenchuan, People's Republic of China
}

\author{
Yi Hu ${ }^{1,2,3 \S}$, Jin-Feng Wang ${ }^{2}$, Xiao-Hong $\mathrm{Li}^{1,45}$, Dan Ren ${ }^{1}$, Dez-Hi Mu${ }^{5}$, Yan-Ping Wang ${ }^{1}$, Chun- \\ Mei Wen ${ }^{6}$, Jun Zhu' ${ }^{1}$, Juan Liang ${ }^{1}$ \\ ${ }^{1}$ National Office for Maternal and Child Health Surveillance, West China Second Hospital, Sichuan University, \\ Chengdu, People's Republic of China; ${ }^{2}$ State Key Laboratory of Resources and Environmental Information \\ System, Institute of Geographic Sciences and Natural Resources Research, Chinese Academy of Sciences, \\ Beijing, People's Republic of China; ${ }^{3}$ School of Earth and Mineral Resource, China University of Geosciences, \\ Beijing, People's Republic of China; ${ }^{4}$ West China School of Public Health, Sichuan University, Chengdu, \\ People's Republic of China; ${ }^{5}$ Department of Pediatrics, West China Second Hospital, Sichuan University, \\ Chengdu, People's Republic of China; ${ }^{6}$ World Health Organization Representative China Office, Beijing, \\ People's Republic of China
}

\begin{abstract}
A Bayesian geostatistical model was used to identify factors related to topographical variation in the analysis of mortality risk for children less than 5 years of age in the 2008 Wenchuan earthquake in the People's Republic of China. Epidemiological data from a standardized survey were available from 115 locations across the study area. Physical and demographic factors measured directly at the township level were examined with respect to risk. The geostatistical model explicitly accounted for spatial correlation present in child mortality by fitting a Gaussian linear model to the data. Results identified the role of several factors in explaining geographical heterogeneity in child mortality and show that it was significantly correlated with earthquake intensity, population density, migrant labour, and the percentage of collapsed houses.
\end{abstract}

Keywords: under-five mortality, risk factors, Bayesian geostatistical model, earthquake, geographical information systems, People's Republic of China.

\section{Introduction}

An earthquake measuring 8.0 on the Richter scale hit Wenchuan county, Sichuan province at 14:28 hours local time on 12 May 2008. Its strength and its catastrophic impact made it among the strongest recorded in Chinese history and also in the world. It caused great damage to an area over $100,000 \mathrm{~km}^{2}$ and was felt in most parts of the country. Sichuan province

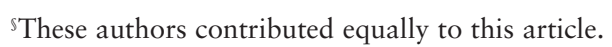

Corresponding author:

Jinfeng Wang

State Key Laboratory of Resources and Environmental Information System

Institute of Geographic Sciences and Natural Resources Research Chinese Academy of Sciences, Beijing, China

Tel. +86 10-64888965; Fax +86 10-64889630

E-mail: wangjf@igsrnr.ac.cn

Juan Liang

National Office for Maternal and Child Health Surveillance

West China Second Hospital, Sichuan University,

Chengdu, Sichuan, China

Tel. +86 28-85501363; Fax +86 28-85501386

E-mail: liangjuan002@163.com with the counties Beichuan, Shifang, Dujiangyan, Mianzhu, Wenchuan and Pengzhou, was the most severely affected area (Liu and Sun, 2009). The devastating earthquake claimed more than 69,000 lives with more than 374,000 people seriously injured, and 18,000 reported missing (Li et al., 2009). Among the large number of dead or missing were many children, particularly those less than 5 years old (Watts, 2008).

Major earthquakes and other catastrophic events can be considered outliers and result from mechanisms involving amplifying critical cascades (Sornette, 2002). It is a growing and urgent need to understand the intermittent dynamics of disasters in order to improve our ways to deal with the negative effects imposed by such occurrences (Tan et al., 2009). Child mortality, particularly in children under 5 years, here referred to as "under-five mortality" is an important indicator of overall health-related vigilance and level of development that should be of concern to policymakers and international organizations aiming to improve public health and living standards. Children under 5 years are largely dependent on their families and lack the ability to protect themselves due to their 
psychological and physical vulnerability, and are therefore more affected by environmental calamities than other age groups. Child mortality is therefore an important indicator of environmental risks associated with earthquakes. Hence, in order to mount the adequate, preventive measures needed, the identification of spatial patterns and epidemiological characteristics of child mortality must come first.

In previous earthquake-related studies (De Bruycker et al., 1985; Armenian et al., 1997; Peek-Asa et al., 1998; Ellidokuz et al., 2005; Gutierrez et al., 2005), epidemiological methods were usually used to assess risk factors. However, the traditional methods do not take into account spatial patterns of occurrences of mortality and injuries and they do not evaluate the risk factors accordingly. Ignoring this correlation results in underestimation of the variance of the effects of risk factors (Cressie, 1993) highlighting the need of developing suitable techniques for incorporating this correlation when assessing the risk of child mortality.

In this study, we use a fully Bayesian geostatistical approach (Diggle et al., 1998) to model the spatial distribution of under-five mortality in the Wenchuan earthquake. It explicitly incorporates the spatial correlation structure of the data, the ability to include covariate effects and the comprehensive representation of uncertainty in the model outputs. Here, the modeling of under-five mortality is measured by the mortality rate at the township level and this approach provides estimates of the effects of various factors influencing mortality patterns including township-level environmental variables.

From an epidemiological point of view, earthquakes can be seen as a "disease" whose mortality can be linked to a number of contributing factors (Gutierrez et al., 2005). To elucidate those contributing effects on child mortality, we collected and analysed the township-specific data of physical and demographic factors. We first identified and mapped the spatial distribution of under-five mortality at the township level and then considered other relevant physical and demographic factors, such as earthquake intensity, population density, and the extent of collapsed houses. Finally, we employed a spatial Gaussian linear model to assess the association between child mortality and those factors.

\section{Materials and methods}

\section{Data of earthquake-induced mortality}

The data included in this study was defined as earthquake-induced deaths among children below 5 years of age, either being part of the local or the floating population (a phrase that describes current unprecedented migration flows, i.e. people moving from underdeveloped areas to more developed areas looking for work and a better life). We distinguished between direct deaths, defined as caused by structural failures such as being struck or trapped by an object dislodged during the earthquake or by falling, and indirect deaths which included deaths caused by fire or secondary shocks in the aftermath of the main earthquake. Death due to traffic control failure and other car accidents were also defined as indirect.

We obtained under-five mortality data collected at the township scale from the National Office for Maternal and Child Health Surveillance (Wang et al., 2011a). A three-level surveillance network was established immediately after the earthquake which was adapted from the national three-level maternal and children surveillance system. This specialized system was composed of interviewers at the county-level recruited from local maternal and child health hospitals in the study areas, interviewers at the townshiplevel recruited from town hospitals, and interviewers at village-level were comprised of village doctors and village chiefs. Most of the interviewers $(80 \%)$ had been engaged in collecting child health data for several years and had valuable and rich experience in this field. Moreover, the village-level interviewers had been living locally for many years and were acquainted with each family in their villages. All the interviewers, however, still received further training on interview techniques.

Up to May 2009, a total of 934 earthquake-related deaths of children less than 5 years, distributed over 115 townships within 21 counties, were confirmed by the National Office for Maternal and Child Surveillance with 683 cases of direct death and 251 cases of indirect death. We calculated township-specific crude mortality rates for each of the 115 townships within a geographical information system (GIS) environment (ArcGIS version 9.3; ESRI, Redlands, CA, USA), which was defined by dividing the number of deceased children by under-five child population, and then multiplying by 1,000. Census data about the under-five population at the township level, however, were lost in most earthquake-hit areas. We obtained the county-level population of the under-five data at the Municipal Civil Affairs Bureau and estimated the township-level population of the under-five $\left(p_{t_{0}}\right)$ as follows:

$$
p_{t_{0}}=p_{c_{0}} \frac{p_{t_{1}}}{p_{c_{1}}},
$$


where $p_{c_{0}}$ is the under-five population at county level, $p_{t_{1}}$ the population at township level and $p_{c_{1}}$ the population at county level.

\section{Risks of under-five mortality}

The physical data included the elevation, the topographic slope, the earthquake intensity and the distance from town to fault. The elevation was obtained using a digital elevation model (DEM) which is a digital model or three-dimensional representation of a terrain surface created from elevation data. The DEM data were derived from the shuttle radar topography mission (SRTM), an international project spearheaded by the U.S. National Geospatial-Intelligence Agency and the U.S. National Aeronautics and Space Administration (http://www2.jpl.nasa.gov/srtm/). The topographic slope, defined by the plane tangent to a topographic surface (Burrough, 1986), was sourced from the DEM as well with the purpose of highlighting the topography of the environment in relation to habitation. We took the topographic slope into account because it is an important factor in landslide hazard analysis, mudslides being prone to start on steep slopes when activated by natural disasters (Chen and Wang, 2009). Indeed, about one third of the all Wenchuan earthquake human losses were not directly due to the earthquake but by the secondary geological events (Liu et al., 2009). Earthquake intensity in the study area was determined using an official Chinese Earthquake Administration Modified Mercalli Intensity (MMI) scale map, which is divided into 12 continuous categories (Wood and Neumann, 1931). The MMI in the study area ranged from VII to XI. There are a series of thrust faults that affect the study area strongly, for example, the Wenchuan-Maoxian, Yingxiu-Beichuan, and Anxian-Guanxian faults (Ye et al., 2008). The earthquake intensity zones were elliptical with those faults as major axes. Townships in the same intensity zone suffered the same devastating power described above, though some were closer to the epicenter. In addition, the rupture of the crust around those faults was the main cause for the structural damage. Consequently, we assumed that studying distance to faults to be more meaningful than studying distance to the epicenter. Using a GIS environment, the distance from town to fault was determined by vertical distance from township to its nearest fault.

Table 1. Range of physical and demographic factors for the 115 townships involved in the study.

\begin{tabular}{|c|c|c|c|c|c|}
\hline Description & Type & Range & Category & Median & Standard deviation \\
\hline Elevation & Continuous & $480-3430 \mathrm{~m}$ & $\begin{array}{c}{[480,500]} \\
(500,1000] \\
(1000,1500] \\
(1500,3430]\end{array}$ & 779 & 51 \\
\hline Topographic slope & Continuous & 0-50.13 degree & $\begin{array}{c}{[0,8]} \\
(8,15] \\
(15,25] \\
(25,50.13]\end{array}$ & 1681 & 1507 \\
\hline Earthquake intensity & Categorical & VII-XI & - & & \\
\hline Distance to fault & Continuous & $0.09-86.78 \mathrm{~km}$ & $\begin{array}{c}{[0.09,1]} \\
(1,5] \\
(5,10] \\
(10,15] \\
(15,86.78]\end{array}$ & 448 & 1189 \\
\hline Population density & Continuous & $3-18,191$ persons $/ \mathrm{km}^{2}$ & $\begin{array}{c}{[3,100]} \\
(100,500] \\
500,1000] \\
1000,18191]\end{array}$ & 200 & 193115 \\
\hline Migrant worker & Continuous & $0-14,698$ persons & $\begin{array}{c}{[0,1000]} \\
(1000,3000] \\
(3000,5000] \\
(5000,14698]\end{array}$ & 1763 & 301644 \\
\hline Per capita income & Continuous & 7-5,377 RMB & $\begin{array}{c}{[7,100]} \\
(100 \sim 300] \\
(300 \sim 500] \\
(500,5377]\end{array}$ & 301 & 57783 \\
\hline Collapsed house & Continuous & $8-94 \%$ & - & 49 & 21 \\
\hline
\end{tabular}


The demographic data include population density, migrant worker, per capita income and information about collapsed houses. "Migrant workers" refers to people who have left their hometowns (within the study area) to look for jobs in more developed areas. The magnitude of the number of collapsed houses was set as the proportion of completely collapsed houses in relation to all registered houses in the area. A completely collapsed building was defined as a structure that could not be repaired but had to be torn down and rebuilt. All the demographic data were obtained from the Evaluation of Bearing Capacity of Resources and Environment (EBCRE) database, which was set up in 2009 by the Institute of Geographic Sciences and Natural Resources Research (IGSNRR), Chinese Academy of Science (CAS), using the national reconstruction plan (Fan, 2009; Wang et al., 2011b).

The management and calculation of the risk variables were implemented using ArcGIS, version 9.3. The ranges of physical and demographic data for the 115 townships are listed in Table 1.

\section{Statistical analysis}

Gaussian regression model was fitted to child mortality using R, version 2.12.0 (Bell Laboratories, Murray Hill, NJ, USA) to identify significant physical and demographic covariates. A Bayesian hierarchical model was fitted to estimate the amount of spatial heterogeneity in child mortality as well as associations between risk factors and child mortality in the presence of spatial correlation. As we mentioned above, ignoring this correlation would underestimate the variance of the effects of risk factors, which would further result in spurious significance of risk factors. We used a Gaussian linear model with a spatially structured, township-specific random effect to assess geographic heterogeneity and the effects of various covariates. The spatial random-effect $(S(\bullet))$ was modeled as a stationary Gaussian process (signal) with the mean 0 , variance $\sigma^{2}$ and correlation function $\rho(u)$, where $u$ is the distance between two townships. The exponential correlation function was used here which is defined as follows:

$$
\rho(u)=\exp (-u / \phi)
$$

in which $\phi>0$ is a scale parameter with the dimensions of distance. Under this function, the practical range (defined as the minimum distance at which spatial correlation between locations is below $5 \%$ ) is approximately $3 \phi$. In addition, a "nugget effect" $(Z)$, which refers to a discontinuity at the origin in the variogram in geostatistical practice, was included in the model out of practical consideration. $Z_{i}(i$ indentifies a twodimensionally spatial location) variables are mutually independent $N\left(0, \tau^{2}\right)$ random variables. Within our model-based framework, $Z_{i}$ can be interpreted as the conditional variance of each measured value $Y_{i}$ (response variable), given the underlying signal value $S\left(x_{i}\right)$. Hence the spatial Gaussian linear model can be summarised as follows:

$$
Y=D \beta-S\left(x_{i}\right)-Z_{i}
$$

which equation can be further expressed as

$$
Y \sim N\left(D \beta, \sigma^{2} R(\phi)+\tau^{2} I\right)
$$

where $D$ is an $n \times p$ ( $n$ and $p$ represent spatial locations and regressors respectively) matrix of covariates $\beta$, is the corresponding vector of regression parameters, $R$ depends on a scalar or vector-valued parameter $\phi$, and $I$ is the identity matrix.

To complete the Bayesian model specification, we need to adopt prior distributions for the model parameters. We chose a conjugate prior in the Gaussian linear model. Specifically, we chose non-informative uniform priors for the regression coefficients, that is, $p(\beta) \propto 1$, and a prior $p\left(\sigma^{2}\right) \propto 1 / \sigma^{2}$ for the signal variance $\sigma^{2}$. For the correlation function parameter $\phi$ we adopted a reciprocal prior $p(\phi) \propto 1 / \phi$. To accommodate a nugget variance $\tau^{2}$, we used a variance ratio $v^{2}=\tau^{2} / \sigma^{2}$ and adopted a uniform prior for $v^{2}$. A discretisation method of equal width intervals was used to discretise prior distributions to render the computations feasible. In the absence of any prior knowledge about the model parameters, the choice of noninformative improper priors should, roughly speaking, reflect our prior guess or practical considerations, e.g. allowing the data to have a greater influence in determining the posterior.

To simulate samples from posteriors, we used a direct Monte Carlo approach, the form of which is direct simulation, replicated independently, rather than Markov chain Monte Carlo algorithm. Hence, issues of convergence do not arise and the simulationinduced variance in sampling from the posterior for any quantity of interest is inversely proportional to the number of simulated replicates (Diggle and Ribeiro, 2007). This provides us an opportunity to assess the magnitude of the simulation-induced variation in the estimated posterior and to adjust the number of simulations if necessary. 
Initial analysis indicated that the distribution of child mortality rate was not a Gaussian distribution, but with a predominance of low values less than 0.030 , such that the mean was 0.019 and the median was 0.006 . This is a common problem in describing the stochastic variation in a physical quantity. One of the simplest ways to extend the Gaussian model is to assume that the model holds after applying a transformation to the original data. For the child mortality rate variable in our study, we used the Box-Cox family (Box and Cox, 1964) to transform the original mortality rate date as follows:

$$
Y^{*}=\left\{\begin{array}{l}
\left(Y^{\lambda}-1\right) / \lambda: \lambda \neq 0 \\
\log Y: \lambda=0
\end{array},\right.
$$

where $Y$ is positive, e.g. $Y>0$. Note that there is no reason why a transformation which stabilizes the variability in the measurements conditional on the signal should also stabilize the variability in the signal, or vice versa (Diggle and Ribeiro, 2007).

The maximum likelihood estimation of $\lambda$ in (4) is -0.10 , e.g. $\lambda^{\wedge}=-0.10$, with its $95 \%$ confidence of $(-0.21,0.01)$. With 0 falling in this confidence, we chose $\lambda=0$, e.g. $\log$ transformation, to the original mortality rate data, and spatial Gaussian linear model (2) can be expressed specifically as follows:

$$
\log Y=\alpha+D \beta+S_{i}+Z_{i}
$$

where $\alpha$ is the intercept, $D$ is a $115 \times 8$ matrix of covariates. A Bayesian non-spatial model was also fitted for comparative purposes which is expressed as follows:

$$
\log Y=\alpha+D \beta+Z^{\prime}
$$

where $Z^{\prime}$ are mutually independent $\mathrm{N}\left(0, \tau^{\prime 2}\right)$ random variables (errors).

Some explanatory variables were categorized, as shown in Table 1 , and used as ordinal variables, and these ordinal variables were treated as quantitative in the model (5). The exception was the percentage of collapsed houses, which was still used as a continuous variable because it has the same scale and significant bivariate correlation $(\gamma=0.65, \mathrm{p}=0.01)$ as the mortality rate.

\section{Results}

We obtained 10,000 samples from posterior distributions and the results of the Bayesian geostatistical model are presented in Table 2 . It can be seen from the posterior interval for $\beta_{8}$ of $(0.017,0.989)$ that the per-

\begin{tabular}{|c|c|c|c|c|c|c|c|c|}
\hline \multirow{3}{*}{ Parameter } & \multicolumn{4}{|c|}{ Spatial model } & \multicolumn{4}{|c|}{ Non-spatial model } \\
\hline & \multirow[b]{2}{*}{$\begin{array}{l}\text { Posterior } \\
\text { Mean }\end{array}$} & \multicolumn{2}{|c|}{ 95\% Bayesian CI } & \multirow[b]{2}{*}{$\begin{array}{l}\text { Posterior } \\
\text { Median }\end{array}$} & \multirow[b]{2}{*}{$\begin{array}{l}\text { Posterior } \\
\text { Mean }\end{array}$} & \multicolumn{2}{|c|}{ 95\% Bayesian CI } & \multirow[b]{2}{*}{$\begin{array}{l}\text { Posterior } \\
\text { Median }\end{array}$} \\
\hline & & $\begin{array}{l}\text { Lower } \\
2.5 \%\end{array}$ & $\begin{array}{l}\text { Upper } \\
97.5 \%\end{array}$ & & & $\begin{array}{l}\text { Lower } \\
2.5 \%\end{array}$ & $\begin{array}{l}\text { Upper } \\
97.5 \%\end{array}$ & \\
\hline Intercept $\left(\beta_{0}\right)$ & -4.545 & -5.647 & -3.434 & -4.546 & -4.511 & -5.612 & -3.399 & -4.507 \\
\hline Elevation $\left(\beta_{1}\right)$ & 0.062 & -0.084 & 0.209 & 0.062 & 0.061 & -0.080 & 0.202 & 0.061 \\
\hline $\begin{array}{l}\text { Topographic } \\
\text { slope }\left(\beta_{2}\right)\end{array}$ & -0.046 & -0.126 & 0.035 & -0.046 & -0.047 & -0.127 & 0.031 & 0.047 \\
\hline $\begin{array}{l}\text { Earthquake } \\
\text { intensity }\left(\beta_{3}\right)\end{array}$ & 0.275 & -0.176 & 0.380 & 0.275 & 0.278 & 0.178 & 0.378 & 0.277 \\
\hline $\begin{array}{l}\text { Distance to } \\
\text { fault }\left(\beta_{4}\right)\end{array}$ & -0.029 & -0.106 & 0.046 & -0.029 & -0.036 & -0.111 & 0.038 & -0.037 \\
\hline $\begin{array}{l}\text { Population } \\
\text { density }\left(\beta_{5}\right)\end{array}$ & -0.130 & -0.250 & -0.008 & -0.129 & -0.126 & -0.245 & -0.009 & -0.126 \\
\hline $\begin{array}{l}\text { Migrant } \\
\text { worker }\left(\beta_{6}\right)\end{array}$ & -0.032 & -0.131 & 0.068 & -0.033 & -0.040 & -0.137 & 0.059 & -0.040 \\
\hline $\begin{array}{l}\text { Per capita } \\
\text { income }\left(\beta_{8}\right)\end{array}$ & 0.019 & -0.091 & 0.112 & 0.019 & 0.017 & -0.073 & 0.112 & 0.018 \\
\hline $\begin{array}{l}\text { Collapsed } \\
\text { house }\left(\beta_{9}\right)\end{array}$ & 0.555 & 0.017 & 0.989 & 0.554 & 0.540 & 0.100 & 0.974 & 0.541 \\
\hline$\sigma^{2}$ & 0.032 & 0.009 & 0.112 & 0.031 & - & - & - & - \\
\hline$\phi$ & 26.47 & 2.00 & 60.00 & 24.00 & - & - & - & - \\
\hline$\tau^{2}\left(\tau^{\prime 2}\right)$ & 0.291 & 0.011 & 2.195 & 0.020 & 0.213 & 0.161 & 0.280 & 0.210 \\
\hline
\end{tabular}

Table 2. Point estimates (posterior means and medians) and $95 \%$ central quantile-based credible intervals (CI) for the parameters of the models fitted to under-five mortality. 
centage of collapsed houses had a strong and significant effect on under-five mortality (relative risk (RR): $\exp (0.555)=1.74 ; 95 \%$ confidential interval $(\mathrm{CI})=$ $1.02,2.69)$, indicating that a $10 \%$ increase in the rate of collapsed houses yields a $6 \%(\exp (0.555 * 0.1=$ 1.06) increase in child mortality risk after adjustment for the other risk factors. The earthquake intensity was also identified as an important and significant risk factor $(\mathrm{RR}: \exp (0.275)=1.32 ; 95 \% \mathrm{CI}=1.19,1.46)$, i.e. a one-level increase in MMI yields a $32 \%$ increase in the mortality risk $(\exp (0.275)=1.32)$ holding other factors. In addition, children living in populated areas were, however, at a significantly lower risk than those living in less populated areas (RR: $\exp (-0.130)=0.88$; $95 \% \mathrm{CI}=0.78,0.99)$. The effects of the remaining variables on child mortality were not significant. The parameter estimates obtained from the non-spatial model are also shown in Table 2. We found that the point estimates of the model coefficients change little but the confidence intervals become wider after taking into account the spatial correlation present in child mortality, confirming the importance of taking spatial correlation into account when analysing geographical data (Cressie, 1993).

Figure 1 displays the distribution of the under-five mortality. A trend of rising mortality starting in the north and running in an easterly direction was observed, which is in line with that of the thrust faults in the area. Different high-mortality risk zones (one around the epicenter, another in the central area and a third in the Northeast) surrounded by low-mortality risk zones, indicating a spatial pattern of alternating high-mortality and low-mortality clusters, can be seen.

The rising trend in the empirical variogram (which was drawn using the geoR package with the variog function) of the transformed under-five mortality in each location in Fig. 2a indicates that spatial correlation is present in child mortality though it is not very strong, and the intercept in this figure justifies the inclusion of a "nugget effect" $Z_{i}$ in the model. The posterior median of 24 for $\phi$ corresponds to the practical range of $3 * 24 \approx 72 \mathrm{~km}$ being the distance at which the correlation reduces to 0.05 . However, the $95 \%$ CI $(2,60)$ of $\phi$ indicates a wide variation of the practical range, from $6 \mathrm{~km}$ to $180 \mathrm{~km}$, probably due to fact that spatial correlation is not very prominent. All the parameters have mostly symmetric posterior distributions excepting $\phi$ and $\tau^{2}$ which are slightly moderately skewed. The empirical variogram of the residuals from the fitted non-spatial regression model in Fig. $2 \mathrm{~b}$ still has a rising trend. This indicates that the non-spatial regression model is inadequate in explaining the spatial variation of the child mortality, whereas the variogram of the residuals from the spatial model shown in Fig. 2c fits the data better though there is still a slightly rising trend.

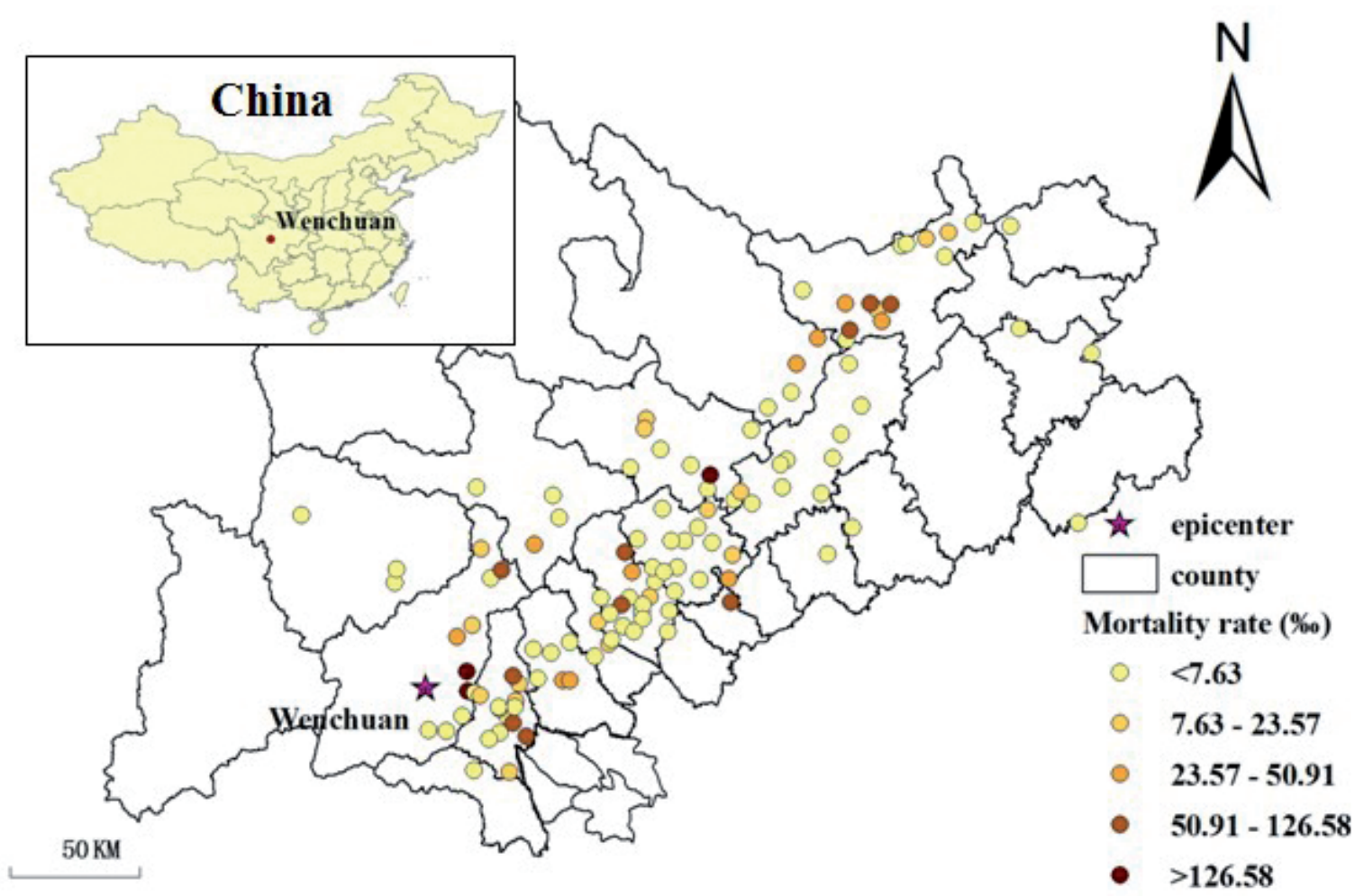

Fig. 1. Under-five mortality rate at the township level. Thematic categories based on the Jenks natural breaks method. 
(a)

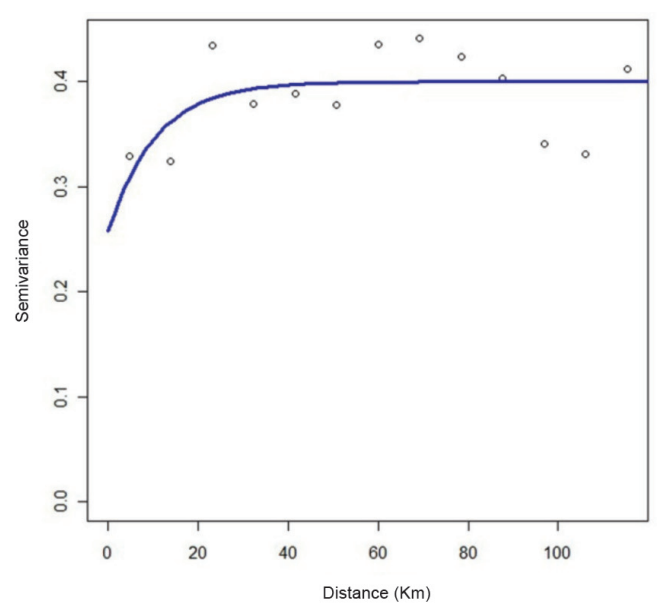

(b)

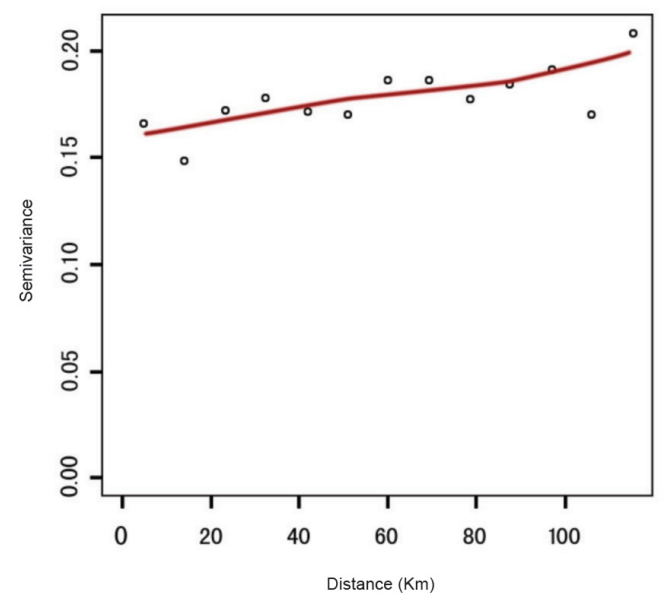

(c)

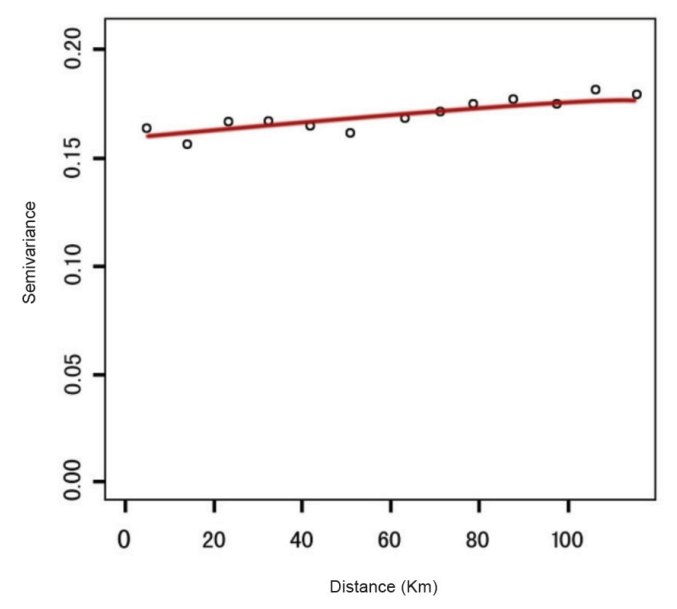

Fig. 2. (a) Empirical variogram of the transformed (log) underfive mortality rate in each location. (b) Empirical variogram of the average of the residuals for each location from the non-spatial model. (c) Empirical variogram of the average of the residuals for each location from the spatial model. The curve in (a) was produced using the exponential function; the curves in (b) and (c) use a loess smoother.

\section{Discussion}

This study presents a novel application of Bayesian geostatistical modeling in assigning risk factors of under-five mortality in the 2008 Wenchuan earthquake. Results identified geographical differences in child mortality and the important role of environmental risk factors in explaining this geographical heterogeneity in terms of earthquake intensity, population density, migrant workers and collapsed houses. The spatial Gaussian linear model explains the spatial variation of child mortality across the study region better than the non-spatial model. This is because the consideration of spatial correlation results in more precise estimates of the standard error and widens the confidence limits of the model coefficients.

Structural factors play an important role in mortality (Tiedemann, 1989; Spence et al., 1990; Coburn et al., 1992). Death and injury in most earthquakes are largely attributed to trauma caused by partial or complete collapse of buildings and infrastructures. In our study area, timber roofs supported either by masonry structures or reinforced masonry structures were widely used in residential buildings, schools in the rural areas, factories and office buildings in the cities (Ye et al., 2008). Many of these buildings collapsed completely or were heavily damaged resulting in lots of direct deaths. Long-term use, lack of maintenance of structural systems and poor connections between precast slabs are generally believed to be main reasons of the vulnerability of these buildings (Ye et al., 2008). Our study agrees with the positive association between earthquake intensity and mortality described previously (Tiedemann, 1989; Alexander, 2000; Gutierrez et al., 2005). The association between population density and mortality has also been reported (Osaki and Minowa, 1999; Gutierrez et al., 2005). However, this association is inconsistent, for example, our study shows child mortality to be negatively associated with population density, thus indicating higher child mortality in less populated zones. The distribution of the population in the study area is uneven with a stronger focus in the East (the plain area) than in the West (the mountainous area). In addition, the economic situation is stronger in the East compared with the areas in the West (Fan, 2009). Hence, there is a high probability that the children living in the less populated and mountainous areas were more vulnerable to the earthquake because of poor living conditions. What is noteworthy is that migrant workers were considered in the model for the reason that they play an important role for the situation of the children. With the rocketing 
development of the People's Republic of China's economy since the 1980 s, labour is badly needed and increasing numbers of rural people, especially young adults, flocked to towns and cities in search of work leaving their children at home. These children became the so-called "left-behind children", usually taken care of by their grandparents. Compared with their elderly grandparents, who are also particularly vulnerable with respect to earthquakes, the parents could have provided greater support and protection, both psychologically and physically, in this emergency. However, the migrant workers were not identified as a significant factor related to child mortality and a specific indepth study, e.g. an individual-level study, needs to be done to account for this association.

Although numerous earthquake-related studies have investigated the relation between risk factors and mortality, to our knowledge, this is the first study attempting to assess this relation by taking into account the geographical variation that is present in assessments of mortality. Evidently, however, the current spatial Gaussian model (5) does not account for anisotropy, which refers to the property of being directionally dependent, and non-stationarity, which is defined as a quality of a process whose mean and standard deviation change with time. Hence, there is a scope for future investigation of this as data are being collected in 21 counties which cover wide areas with marked environmental diversity. The somewhat rising trend of the residual variogram in Fig. $2 \mathrm{c}$ is probably due to lack of risk factors characterizing child mortality. This indicates that more risks should be considered in further studies, such as access to major transportation routes and major medical centers which are closely related to emergency services. In addition, ecological studies cannot control for the effects of confounding factors. This study is therefore open to the risk of the ecological fallacy (Washio et al., 2008), i.e. taking the finding of a linear relationship between exposure and outcome as evidence that this relation should also hold at the individual level. Then again, our approach is appropriate for hypothesis generation and provides essential needs information providing a base for a more rigorous study design of child mortality, e.g. an individual-level study. Furthermore, for each categorized explanatory variable a linear effect (one beta coefficient for such categorized covariates) was assumed in equation (5), and this may disguise, possibly, the non-linear effect between these covariates and child mortality. However, it is advantageous to treat these covariates in a quantitative manner as equation (5) fits relatively well; for example, this model is sim- pler and easier to interpret, and it is more convenient to draw empirical variogram of residuals with such parsimonious model. Finally, the validation of cases of child mortality was questionnaire-based. Because the families of some of the deceased children, including relatives and neighbours, died in the earthquake as well, information related to how and where they died was unclear. However, to avoid missing and fault reports, the National Office for Maternal and Child Health Surveillance tried hard to search for villagers who might know more about these deceased children.

Despite these limitations, we believe that this study is useful because it focuses on an area of public attention where relatively little is known about cause and effect. In addition, because it demonstrated the potential of spatial statistical methods for analysing mortality data in disasters and, perhaps most importantly, because it helps researchers to understand the spatial patterns in child mortality encouraging further study.

\section{Acknowledgements}

This research was funded by CAS (XDA05090102), NSFC (41023010), the MOST (2012CB955500; 2011AA120305; 2012ZX10004-201), and Program for Changjiang Scholars and Innovative Research Team in University (IRT0935).

\section{References}

Alexander D, 2000. On the spatial pattern of casualties in earthquakes. Ann Epidemiol 10, 1-4.

Armenian HK, Melkonian A, Noji EK, Hovanesian AP, 1997. Deaths and injuries due to the earthquake in Armenia: a cohort approach. Int J Epidemiol 26, 806-813.

Box GEP, Cox DR, 1964. An analysis of transformations (with discussion). J Roy Stat Soc B 26, 211-252.

Burrough PA, 1986. Principles of geographical information systems for land resources assessment, Clarendon Press, 123 pp.

Chen CT, Wang TT, 2009. New slope indexes for landslide potential analysis in debris flow Stream. Geophys Res Abstr 11,5615 .

Coburn AW, Spence RJS, Pomonis A, 1992. Factors determining human casualty levels in earthquakes: mortality prediction in building collapse. Proceedings of the Tenth World Conference on Earthquake Engineering 10, 5989-5994.

Cressie N, 1993. Statistics for spatial data. John Wiley \& Sons, Inc, $240 \mathrm{pp}$.

De Bruycker M, Greco D, Lechat MF, Annino I, De Ruggiero N, Triassi M, 1985. The 1980 earthquake in Southern Italymorbidity and mortality. Int J Epidemiol 14, 113-117.

Diggle PJ, Ribeiro PJ, 2007. Model-based geostatistics. Springer, $51 \mathrm{pp}$ 
Diggle P, Tawn JA, Moyeed R, 1998. Model-based geostatistics. Appl Stat 47, 299-350.

Ellidokuz H, Ucku R, Aydin UY, Ellidokuz E, 2005. Risk factors for death and injuries in earthquake: cross-sectional study from Afyon, Turkey. Croat Med J 46, 613-618.

Fan J, 2009. Evaluation of bearing capacity of resources and environment. Beijing, China. Science Press, 133-136 pp.

Gutierrez E, Taucer F, De Groeve T, 2005. Analysis of worldwide earthquake mortality using multivariate demographic and seismic data. Am J Epidemiol 161, 1151-1158.

Li S, Rao LL, Ren XP, Bai XW, Zheng R, Li JZ, Wang ZJ, Liu H, 2009. Psychological typhoon eye in the 2008 Wenchuan earthquake. PLoS One 4, e4964.

Liu Z, Sun S, 2009. The disaster of May 12th Wenchuan earthquake and its influence on debris flows. J Geogr Geol 1, 26-30.

Osaki Y, Minowa M, 1999. Factors associated with earthquake deaths in the great Hanshin-Awaji earthquake, 1995. Am J Epidemiol 46, 175-183.

Peek-Asa C, Kraus J, Bourque L, Vimalachandra D, Yu J, Abrams J, 1998. Fatal and hospitalized injuries resulting from the 1994 Northbridge earthquake. Int J Epidemiol 27, 456-465.

Sornette D, 2002. Predictability of catastrophic events: material rupture, earthquakes, turbulence, financial crashes, and human birth. Proc Natl Acad Sci USA 99, 2522-2529.

Spence RJS, Coburn AW, Sakai S, 1990. Reducing human casualties in building collapse: methods of optimizing disaster plans to reduce injury levels. Martin Center for Architectural and Urban Studies, Cambridge University, 55 pp.
Tan CE, Li HJ, Zhang XG, Zhang H, Han PY, An Q, Ding WJ, Wang MQ, 2009. The impact of the Wenchuan earthquake on birth outcomes. PLoS One 4, e8200.

Tiedemann H, 1989. Casualties as a function of building quality and earthquake intensity. Proceedings of the International Workshop on Earthquake Injury Epidemiology for Mitigation and Response, Johns Hopkins University, 420-434.

Wang JF, Liao YL, Wang JJ, Chen T, Fang J, Gao XL, Cao ZD, Hu MG, Ge Y, Zheng XY, 2011b. Data adaptive modeling human-environment relationship and its application in displacing the quake population. Popul Environ. DOI: 10.1007/s11111-011-0143-3.

Wang YP, Miao L, Dai L, Zhou GX, He CH, Zhu J, Liang J, 2011a. Mortality rate for children under 5 years of age in China from 1996 to 2006. Public Health 125, 301-307.

Washio M, Oura A, Mori M, 2008. Ecological studies on influenza infection and the effect of vaccination: their advantages and limitations. Vaccine 26, 6470-6472.

Watts J, 2008. Chinese quake forced $3 \mathrm{~m}$ children from homes, Vol. 2009: 2008-2023. http://www.guardian.co.uk/world/2008 /may/23/chinaearthquake.china http://www.guardian.co.uk /world/2008/may/23/chinaearthquake.china (accessed on May 2008).

Wood HO, Neumann F, 1931. Modified Mercalli intensity scale of 1931. B Seismol Soc Am 21, 277-283.

Ye L, Lu X, Zhe Q, Peng F, 2008. Analysis on building seismic damage in the Wenchuan earthquake, The 14th World Conference on Earthquake Engineering. Beijing, China. 